\title{
Subxiphoid single-incision thoracoscopic left upper lobectomy
}

\author{
Chia-Chuan Liu, MD, ${ }^{\mathrm{a}}$ Bing-Yen Wang, MD, ${ }^{\mathrm{b}, \mathrm{c}}$ Chih-Shiun Shih, MD, ${ }^{\mathrm{a}}$ and Yun-Hen Liu, MD, ${ }^{\mathrm{d}}$ Taipei, \\ Taichung, and Keelung, Taiwan
}

Thoracoscopic surgery has been shown to be a feasible and safe technique for performing lobectomy. ${ }^{1}$ Thoracoscopic lobectomy is normally performed though 2 or 4 incisions, although cases of single-incision thoracoscopic lobectomy have been reported, ${ }^{2,3}$ We present a novel technique for performing thoracoscopic lobectomy through a single subxiphoid incision.

\section{CLINICAL SUMMARY}

A 49-year-old woman came to our clinic with a pulmonary nodule that was incidentally found on plain film radiographs of the chest. Computed tomography of the chest demonstrated an irregular soft-tissue lesion, measuring $3 \times 3 \mathrm{~cm}$, in the left upper lobe without mediastinal lymph node enlargement. Positron emission tomography and computed tomography revealed intense focal fludeoxyglu-

From the Division of Thoracic Surgery, ${ }^{a}$ Department of Surgery, Koo Foundation Sun Yat-Sen Cancer Center, Taipei, Taiwan; the Division of Thoracic Surgery, ${ }^{\mathrm{b}}$ Department of Surgery, Changhua Christian Hospital and Institute of Medicine, Chung Shan Medical University, Chung, Taichung, Taiwan; the Division of Thoracic Surgery, ${ }^{\mathrm{c}}$ Department of Surgery, Taipei Veterans General Hospital and National Yang-Ming University School of Medicine, Taipei, Taiwan; and the Division of Thoracic Surgery, ${ }^{\mathrm{d}}$ Chang Gung Memorial Hospital, College of Medicine, Chang Gung University, Keelung, Taiwan.

Disclosures: Authors have nothing to disclose with regard to commercial support.

C.-C.L. and B.-Y.W. contributed equally to this work, and each should be considered first author.

Received for publication Feb 18, 2014; revisions received June 28, 2014; accepted for publication Aug 18, 2014; available ahead of print Sept 17, 2014.

Address for reprints: Chia-Chuan Liu, MD, Division of Thoracic Surgery, Department of Surgery, Koo Foundation Sun Yat-Sen Cancer Center, 125 Lih-Der Rd, Pei-Tou District, Taipei, Taiwan (E-mail: 156283@cch.org.tw).

J Thorac Cardiovasc Surg 2014;148:3250-1

$0022-5223 / \$ 36.00$

Copyright $($ C 2014 by The American Association for Thoracic Surgery

http://dx.doi.org/10.1016/j.jtcvs.2014.08.033 cose (F18) (INN fludeoxyglucose [18F]) uptake in the lesion without distant metastases. Computed tomographically guided biopsy of the mass lesion was performed, and the results of histopathologic analysis of the biopsy specimen were positive for adenocarcinoma, clinical stage T1N0M0.

After written, informed consent was obtained from the patient, a thoracoscopic lobectomy was performed through a small subxiphoid incision. Radical mediastinal lymph node sampling was also carried out. The intensity of postoperative pain was evaluated every 8 hours by means of a visual analog scale, which ranged from 0 (painless) to 10 (worst pain ever experienced). The mean pain scores at rest were 3,2, and 0 immediately after the operation, the day after the operation, and 2 days after the procedure, respectively. Pain was limited to the subxiphoid area. The patient's postoperative course was uneventful, and she was discharged on the third day after surgery.

The resected tumor measured $3.5 \mathrm{~cm}$, and the pathologic stage was T2aN1M0. A total of 15 lymph nodes ( 2 at station $4 \mathrm{~L}, 3$ at station 5, 3 at station 7, 1 at station 10,1 at station 11 , and 5 at station 12/13/14) were dissected, and 2 segmental lymph nodes had positive results for malignancy.

\section{TECHNIQUE}

After induction of general anesthesia and intubation with a double-lumen endotracheal tube, the patient was put in the right semidecubitus position at a $45^{\circ}$ angle to the table. A 4 $\mathrm{cm}$ transverse incision was made over the subxiphoid area, a subcostal tunnel was created, and a wound protector was placed into the wound to provide optimal exposure. The sternocostal margin was lifted with a retractor (Figure 1,

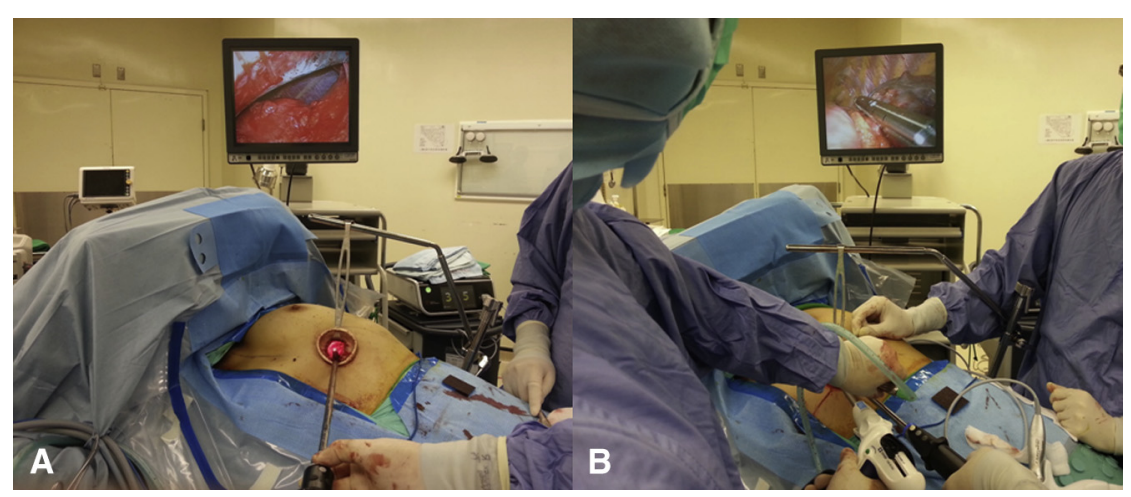

FIGURE 1. A, Photograph of the lifting of the sternocostal margin with a retractor. B, Photograph of the 10-mm, $30^{\circ}$ angled thoracoscope, the endostapler, and other working instruments used during surgery. 


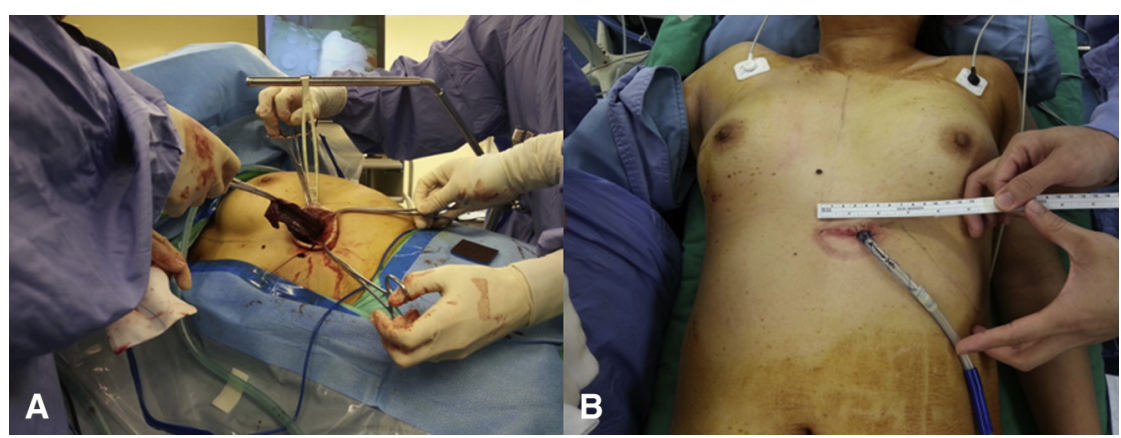

FIGURE 2. A, The lung specimen was placed in a plastic bag and withdrawn through the subxiphoid incision. B, A $24 \mathrm{~F}$ straight chest tube was placed at the left side of the wound.

A). A $10-\mathrm{mm}, 30^{\circ}$ angled thoracoscope was used during the operation.

Gross examination revealed a complete interlobar fissure. The lingular pulmonary and posterior segmental arteries were transected with endoscopic staplers. The superior pulmonary vein was looped and then cut with an endocutter. The anterior segmental pulmonary artery and the apical segmental pulmonary artery were then divided with an endocutter. Finally, the left upper lobe bronchus was transected. We used an Endo GIA articulating endoscopic stapler (Covidien Ltd, Dublin, Ireland) for vascular and bronchial transection. When the endoscopic stapler was applied, the camera's position had to be changed to accommodate the stapler (Figure 1, B). The lung specimen was secured in a protective bag, and the tumor was withdrawn through the subxiphoid incision (Figure 2, A). At the end of surgery, a $24 \mathrm{~F}$ straight chest tube was placed into the left side of the wound (Figure 2, B). The total operative time was 204 minutes, and the volume of blood loss was $30 \mathrm{~mL}$.

\section{DISCUSSION}

We present here the surgical details and preliminary results of single-incision thoracoscopic lobectomy and segmentectomy. Our team is familiar with single-incision thoracoscopic lobectomy through the intercostal approach. ${ }^{3}$ In this case, we made the incision more inferiorly to the subxiphoid area, and the thoracoscopic view was similar to that in the sixth intercostal approach. Some drawbacks of the subxiphoid approach exist. For example, the subxiphoid approach provides limited access to posterior anatomy and makes it difficult to perform complete radical lymph node dissection in the subcarinal area. In our case, we only performed mediastinal lymph node sampling.

The reported incidence of chronic postthoracotomy pain ranges from $11 \%$ to $80 \%,{ }^{4}$ and there is no difference in chronic postoperative pain occurrence between endoscopic and open thoracotomy. Chronic chest wound pain is due to damage to or compression of intercostal nerves, and intercostal incisions in thoracoscopic surgery inevitably cause some intercostal nerve injury. Our technique involves making a single incision in the subxiphoid area and creating a tunnel into the thorax. The technique does not involve intercostal incisions, thereby avoiding potential injury to the intercostal nerves and subsequent intercostal neuralgia. Our findings are based on the results of a single operation, however, and thus no conclusions can be made regarding the effectiveness of our method for the excision of other lobes.

\section{CONCLUSIONS}

Thoracoscopic lobectomy performed through a single subxiphoid incision is a novel approach.

\section{References}

1. Paul S, Altorki NK, Sheng S, Lee PC, Harpole DH, Onaitis MW, et al. Thoracoscopic lobectomy is associated with lower morbidity than open lobectomy: a propensity-matched analysis from the STS database. J Thorac Cardiovasc Surg. 2010;139:366-78.

2. Gonzalez-Rivas D, Paradela M, Fieira E, Velasco C. Single-incision video-assisted thoracoscopic lobectomy: initial results. J Thorac Cardiovasc Surg. 2012;143: $745-7$.

3. Wang BY, Tu CC, Liu CY, Shih CS, Liu CC. Single-incision thoracoscopic lobectomy and segmentectomy with radical lymph node dissection. Ann Thorac Surg. 2013;96:977-82.

4. Rogers ML, Duffy JP. Surgical aspects of chronic post-thoracotomy pain. Eur J Cardiothorac Surg. 2000;18:711-6. 